Journal of Patient-Centered

\title{
The Needs of Women Treated for Ovarian Cancer: Results From a \#gyncsm Twitter Chat
}

\author{
Teresa Hagan Thomas \\ Karin Nauth-Shelley \\ Michael A. Thompson \\ Deanna J. Attai \\ Matthew S. Katz \\ David Graham \\ Dee Sparacio \\ Christina Lizaso \\ Audun Utengen \\ Don S. Dizon
}

Follow this and additional works at: https://aah.org/jpcrr

Part of the Neoplasms Commons, Obstetrics and Gynecology Commons, Oncology Commons, Public Health Education and Promotion Commons, Urogenital System Commons, and the Women's Health Commons

\section{Recommended Citation}

Hagan Thomas T, Nauth-Shelley K, Thompson MA, Attai DJ, Katz MS, Graham D, Sparacio D, Lizaso C, Utengen A, Dizon DS. The needs of women treated for ovarian cancer: results from a \#gyncsm Twitter chat. J Patient Cent Res Rev. 2018;5:149-57. doi: 10.17294/2330-0698.1592

Published quarterly by Midwest-based health system Advocate Aurora Health and indexed in PubMed Central, the Journal of Patient-Centered Research and Reviews (JPCRR) is an open access, peer-reviewed medical journal focused on disseminating scholarly works devoted to improving patient-centered care practices, health outcomes, and the patient experience. 


\title{
The Needs of Women Treated for Ovarian Cancer: Results From a \#gyncsm Twitter Chat
}

\author{
Teresa Hagan Thomas, PhD, RN, ${ }^{1}$ Karin Nauth-Shelley, MBA, ${ }^{2}$ Michael A. Thompson, MD, PhD, ${ }^{3}$ \\ Deanna J. Attai, MD, ${ }^{4}$ Matthew S. Katz, MD, ${ }^{5}$ David Graham, MD, ${ }^{6}$ Dee Sparacio, MS, ${ }^{7}$ Christina \\ Lizaso, MNO, ${ }^{7}$ Audun Utengen, MBA, ${ }^{8}$ Don S. Dizon, MD $^{9}$ \\ ${ }^{1}$ University of Pittsburgh School of Nursing, Pittsburgh, PA; ${ }^{2}$ ThoughtBoost, Boston, MA; ${ }^{3}$ Aurora Research Institute, \\ Aurora Health Care, Milwaukee, WI; ' University of California, Los Angeles, Los Angeles, CA; ${ }^{5}$ Lowell General Hospital, \\ Lowell, MA; ${ }^{6}$ Levine Cancer Institute-Mallard Creek, Charlotte, NC; ${ }^{7}$ \#gyncsm online community; ${ }^{8}$ Symplur, Los \\ Angeles, CA; ${ }^{9}$ Lifespan Cancer Institute, Rhode Island Hospital, Providence, RI
}

Purpose Ovarian cancer is the most fatal of all gynecologic cancers, with a high relapse rate regardless of stage. Women treated for ovarian cancer, therefore, likely have supportive care needs that extend well beyond the time frame of first-line therapy. Unfortunately, there is minimal data describing these needs. The purpose of this qualitative study is to understand the supportive care needs of women with ovarian care at the end of treatment.

Methods To better understand the issues faced by women with ovarian cancer, we conducted a public Twitter chat in collaboration with gynecologic cancer social media (\#gyncsm). Both quantitative and qualitative analyses were performed.

Results The chat occurred over a 1-hour time frame on Twitter and resulted in more than 300 unique and original tweets from 43 participants during the chat and an additional 60 unique participants following the chat. Survivors and physicians represented $32 \%$ and $11 \%$ of participants, respectively; caregivers, advocates, and other clinicians represented the remaining participants. Participants noted deep interest in receiving support during survivorship and dissatisfaction with currently available resources. Sentiment analysis showed that participants viewed the support from social media in a positive light and also revealed negative sentiment around the lack of support from health care providers at the end of treatment.

Conclusions Themes derived from the Twitter chat revealed the unique experiences of individuals with ovarian cancer after treatment, including a heightened sense of vulnerability. Understanding these themes represents an opportunity for clinicians to better understand and address the needs of this patient community. (J Patient Cent Res Rev. 2018;5:149-157.)

Keywords ovarian neoplasm; survivorship care plan; cancer survivorship; patient needs; Twitter

$\mathrm{I}$ ndividuals who complete treatment for ovarian cancer are known to experience a high number of unaddressed needs. ${ }^{1,2}$ Ovarian cancer is also the fifth deadliest cancer for adult women, with an $80 \%$ recurrence rate within the first 2 years after ending primary treatment. ${ }^{3}$ While advanced treatment

Correspondence: Teresa Hagan Thomas, PhD, RN,

University of Pittsburgh School of Nursing, 3500 Victoria Street, 440 Victoria Building,

Pittsburgh, PA 15261 (t.thomas@pitt.edu) modalities have increased survival rates for patients with more limited disease, the supportive needs of patients continue well beyond active cancer treatment. ${ }^{4-6}$

In 2006, the Institute of Medicine recommended that every patient completing primary treatment receive a "comprehensive care summary and follow-up plan."” Despite recommendations for survivorship care plans to summarize participants' treatments, ongoing therapy, follow-up visits, cancer screening and surveillance, late- and long-term effects, and psychosocial concerns, 
this is far from routinely implemented. The uptake of survivorship care plans remains challenging given lack of consensus about data elements to be included in follow-up plans, timing, heterogeneous disease trajectories across and within cancer types, increased (and often unpaid) time required to create and distribute survivorship care plans, and lack of evidence concerning the benefits of these plans. ${ }^{8}$ Although intended to improve patient-provider communication and facilitate shared decision-making at the end of treatment, the difficulty in implementing survivorship care plans has prevented patients from seeing the benefits of these plans.

Patients' supportive needs at the end of treatment remain largely unknown by clinicians and researchers alike. ${ }^{9-11}$ However, identifying these needs is critical to ensuring survivorship care plans fully address the informational, physical, emotional, and support requirements of the ovarian cancer patient population. By understanding patient needs, clinicians can proactively provide resources that increase both quality of life and health during their survivorship experience and assist individuals in earlier recognition of recurrence or late-term treatment effects.

Twitter (Twitter, Inc., San Francisco, CA) is a microblogging social media platform in which people with an account can initiate, discuss, and exchange ideas. Due to its popularity as a mechanism to share and exchange ideas, Twitter attracts lay and professional individuals to engage with each other to share pertinent topics. ${ }^{12}$ Participants share their ideas in the form of a "tweet," which is limited to $\sim 140$ characters. A growing number of cancer patients are finding supportive communities on Twitter, including gynecologic cancer patients. The \#gyncsm (gynecologic cancer social media) hashtag identifies tweets of interest to those whose lives have been impacted by a gynecologic cancer. The hashtag is also used for monthly Twitter chats, which cover educational and patient-focused topics. Health care providers, including one author of this paper (D.S.D.), regularly participate as health care moderators in these chats to provide education and resources to patient groups.

\section{METHODS}

We conducted a digital content analysis of a moderated online discussion forum using the social media platform Twitter, accessing a diverse group of stakeholders knowledgeable about the needs of patients with ovarian cancer. This online discussion was conducted as a regularly scheduled \#gyncsm Twitter chat titled "Re-envisioning Ovarian Cancer Survivorship." The chat was advertised to this active, public social media community (\#gyncsm) so we could obtain a broader perspective of the needs of individuals with ovarian cancer in the survivorship period of their disease. We publicized the discussion, through Twitter, to followers of the Ovarian Cancer Research Fund Alliance, Society for Gynecological Oncology, Foundation for Women's Cancer, and the National Ovarian Cancer Coalition.

The Twitter chat posed 5 questions, developed by the moderators and approved by the authors, to participants (Table 1). To engage participants in deep yet targeted

Table 1. Twitter Chat Questions

\begin{tabular}{|c|c|}
\hline Order & Question \\
\hline $\mathrm{T} 1$ & $\begin{array}{l}\text { A. What does survivorship mean to you? What is it to be an ovarian cancer survivor? } \\
\text { B. Do you use the term survivor? If not, what term do you prefer? }\end{array}$ \\
\hline $\mathrm{T} 2$ & $\begin{array}{l}\text { What needs and concerns did you have when you were first diagnosed and treated? How were they addressed? } \\
\text { What was lacking? }\end{array}$ \\
\hline T3 & $\begin{array}{l}\text { How was the topic of recurrence addressed with you? Did you find it helpful or not helpful at the time - and now } \\
\text { looking back? }\end{array}$ \\
\hline $\mathrm{T} 4$ & $\begin{array}{l}\text { A. What issues - physical, emotional or other - currently give you the most difficulty? } \\
\text { B. What are your needs and concerns now (after recurrence, or, as you live past diagnosis and initial treatment } \\
\text { of ovarian cancer)? }\end{array}$ \\
\hline T5 & $\begin{array}{l}\text { What actions have you taken in living past your ovarian cancer diagnosis and treatment? Were you given a } \\
\text { survivorship care plan? }\end{array}$ \\
\hline
\end{tabular}


discussion, we developed open-ended questions that elicited participant experiences of survivorship including their needs, supportive care received, and unmet needs. Participants were invited to respond to each question and asked to tag their specific tweets to the topic or question to which they were responding (T1A, T1B, T2, etc). Survivors, if comfortable doing so, were invited to identify the year they were diagnosed and their primary cancer type and stage. The Twitter chat moderator reinforced that the \#gyncsm hashtag and the Twitter chat were not forums for medical advice. Authors (T.H.T, D.S.D) also moderated the Twitter chat to ensure the accuracy and appropriateness of all messages. At the beginning and end of the Twitter chat, participants were provided with online resources and supportive services for ovarian cancer survivors.

A mixture of qualitative and quantitative data analysis tools was used to assess participant responses. First, we performed a quantitative analysis of the words used by patients using Symplur Signals (Los Angeles, CA), a company specializing in health care social media analytics. The company's algorithm for assessing word sentiment is based on a scaling system of neutral, positive, and negative words used in health care conversations on Twitter (more details can be found at https://help.symplur.com/reports/content/ sentiment). Unlike traditional qualitative analyses, evaluating patients' sentiments allowed us to assess the overarching disposition of tone using rigorous methods included in this software.

Second, we qualitatively analyzed participant responses to the 5 Twitter chat questions to extract common ideas across participants. Descriptive content analysis methodologies were applied with the goal of organizing participant responses and reducing the data to common themes. ${ }^{13}$ Participant descriptions of their survivorship experiences were summarized and interpreted to create shared narratives and identify unmet needs and barriers to care.

Because this study was mostly interested in the selfreported needs and preferences of survivors, we prioritized tweets from the 15 apparent patients above tweets from providers, caregivers, and advocates in order to focus on those with first-hand experience of ovarian cancer. The first author, who is trained in qualitative data analysis and rigor, reviewed all tweets and provided basic descriptions for initial review by K.N. and for validation by D.S.D. Once confirmed, the codes were applied to all participant responses and summarized with general themes that were confirmed by all study team members. We also used basic descriptive statistics to describe the 1) number of tweets, 2) number of retweets (original tweets that are then shared by others), 3) most frequently used words, and 4) sentiment of frequently used words (either positive or negative).

This study was conducted using a social media platform in which participant responses are publicly available. Therefore, the institutional review board did not require human subjects approval because the analysis only included tweets shared with the \#gyncsm hashtag that are not intended to be private, no individual interaction occurred between participants and the research team, and Twitter's privacy policy explicitly states that all information shared within its website is intended to be broadly shared and that users had minimal expectations for privacy while using the website. ${ }^{14}$ The chat leaders noted at the beginning of the event that information shared with the \#gyncsm would be publicly available, offered participants the opportunity to "listen" only if they were uncomfortable sharing, and referred participants to a disclaimer explaining privacy concerns and medical information (http:// gyncsm.blogspot.com/p/tweet-disclaimer.html).

\section{RESULTS}

The chat occurred on April 13, 2016, from 9 to 10 p.m. Eastern Daylight Time, the usual Twitter chat time for the \#gyncsm community. We allotted 10 minutes to discuss each of the 5 questions. The \#gyncsm leaders initiated each question, and participants responded in real time to each question, though some participants answered questions at a later time.

\section{Quantitative Analysis}

The chat included 377 unique and original tweets and had postings from 43 stakeholder participants during and immediately after the Twitter chat (total: 1 hour, 15 minutes), with 60 additional participants adding to the conversation in the day following the Twitter chat. Among participants, 32\% identified as survivors and $10.7 \%$ were physicians. Other participants included family caregivers, patient advocates, and other clinicians such as social workers. Each participant posted an average of 8.64 tweets. 
The word bubbles in Figure 1 illustrate the most commonly stated words during the Twitter chat. The largest bubbles reflect descriptive words, such as "cancer," "ovarian," and "survivorship," that reflected the topic of conversation. Notably, words such as "support," "lacking," "living," and "advocate" align with participants' statements indicating dissatisfaction with the currently available health care services for ovarian cancer survivors. They indicated the need for additional support to live full, meaningful and healthy lives.

Figure 2 compares the word frequency of positive and negative sentiment words used during the Twitter chat. Overall, participants used words with a positive sentiment more frequently than words with a negative sentiment, though most of the positive words were general in nature (eg, "good," "welcome," and "join"). Remarkable positive sentiment words like "thanks/ thank," "support," and "care" reflected participants' discussion of survivors' needs and their thanks for support they felt from the Twitter chat and caregivers.

By comparison, words with a negative sentiment were much more striking. While prompted to discuss their unmet needs, the tone of participants' word choices reflected the neglect they sensed from their health care providers and the fear they felt while ending treatment. "Struggle," "loss," "panic," and "scary" paint a picture in which survivors feel abandoned in the midst of an intense fight, potentially reflecting the fear and worry survivors stated having when treatment ends and the sense of control and connection with their health care providers is broken.

\section{Qualitative Analysis}

Common themes expressed by participants in the Twitter chat described feeling lost after initial treatment, with continued and new symptoms and side effects from treatment. Women expressed that while

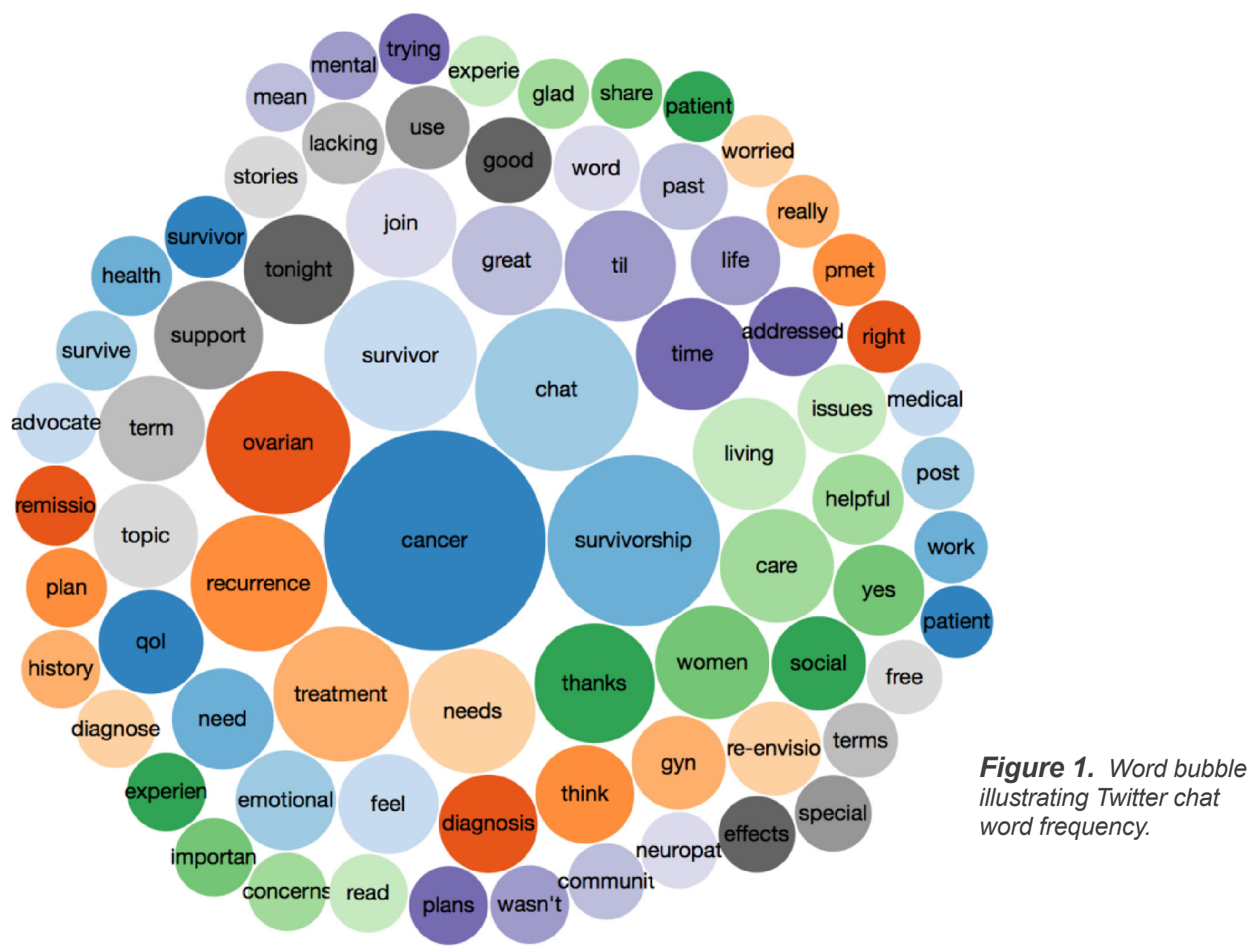




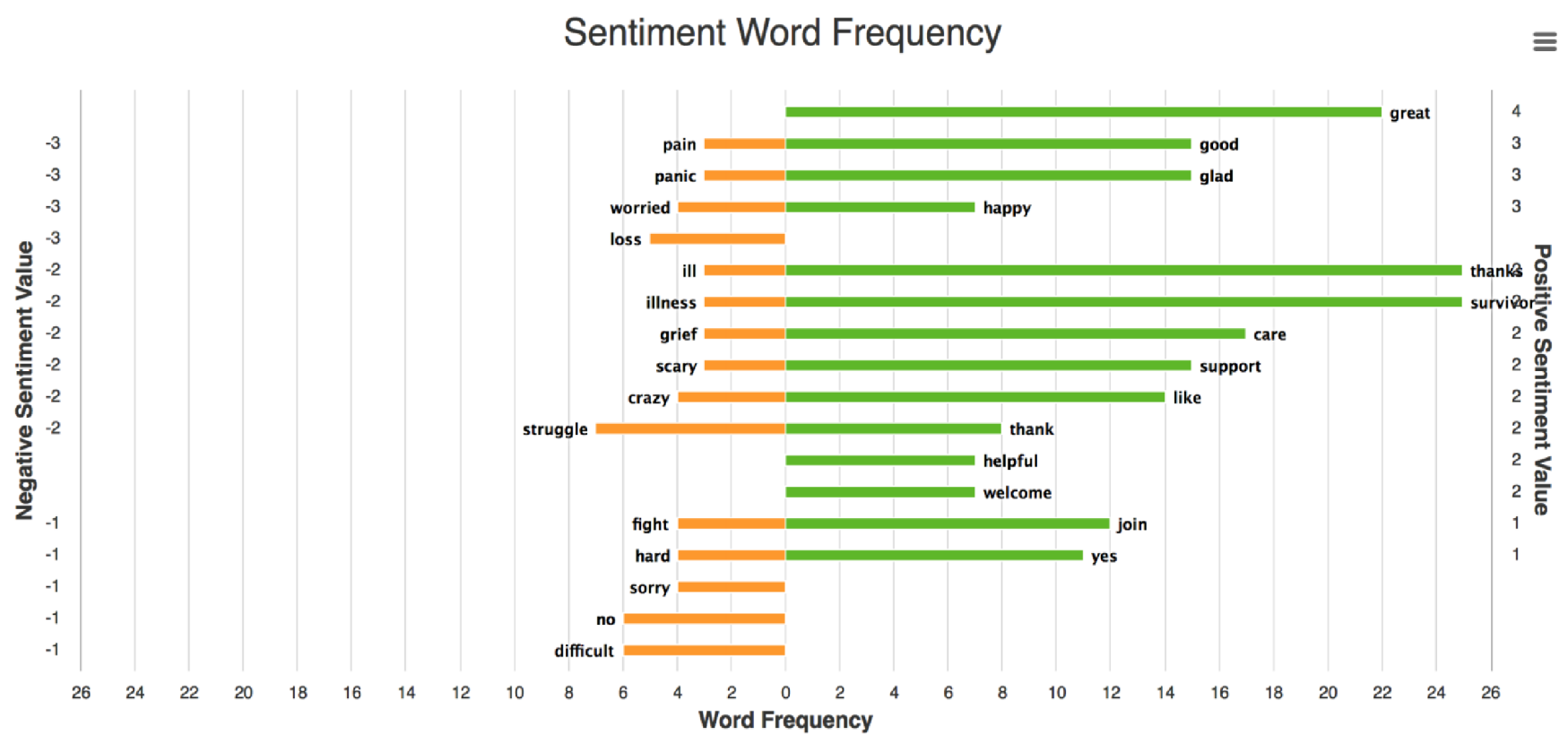

Figure 2. Word frequency by positive and negative sentiment.

they had practical concerns during treatment, their concerns transitioned to becoming more emotional ones in the remission phase. Moreover, few survivors reported receiving a survivorship care plan from their oncology team, and many described a lack of guidance concerning their fears of recurrence, quality-of-life concerns, and symptoms such as neuropathy, memory problems, anxiety, and sleep.

Table 2 lists quotations from the chat representative of common concerns experienced by women with ovarian cancer going off active treatment. Participants defined their period of survivorship as a time in which they focused on attempting to live well and regain the quality of life they lost during treatment. Nonetheless, they reported concerns related to loss of control, uncertainty, and the possibility their cancer could recur. Most women preferred not to be called a "survivor," some suggesting the term "thriver" or someone "in remission" rather than someone who is completely finished with their treatment. Most agreed that the way in which a survivor defines herself should be personally defined rather than imposed on them from others.

While undergoing treatment, survivors recalled their needs as centering around practical concerns, acute symptoms, accessing support and patient stories, and receiving referrals to additional support services.

Participant A: "Most concerned about chemo \& vomiting."

Participant B: "Women seem to seek first to know they are not alone and also practical concerns that come with treatment."

Participant C: "Ifeel what is missing is more stories of women living with OC. When I was first diagnosed I was sure I would not survive."

Table 2. Selected Quotations From Twitter Chat

\begin{tabular}{lc}
\hline Quotation & Retweets \\
\hline $\begin{array}{l}\text { Survivorship is more about living than } \\
\text { surviving. }\end{array}$ & 8 \\
$\begin{array}{l}\text { Recurrence is such a hard topic, but l've } \\
\text { read too many stories of women who felt }\end{array}$ & 7 \\
slammed because it wasn't discussed at all. & \\
$\begin{array}{l}\text { We still have work to do to improve } \\
\text { gynecology patient education on side } \\
\text { effects, emotional needs. }\end{array}$ & 6 \\
\hline
\end{tabular}


In terms of their current concerns, survivors reported having continued symptom management needs, especially for anxiety, sleep concerns, memory problems, and peripheral neuropathy. They also reported significant worries about their cancer coming back and regarding end-of-life issues: "Too much avoidance [among health care providers] of endof-life discussions when needed, death still taboo topic" (Participant D). Symptoms gave survivors the most difficulty, along with managing the possibility of a recurrence. A couple of participants mentioned that guidelines for detecting a recurrence were often unclear and could lead to aggressive testing.

Participant E: "Need clear guidelines [for] longterm remission after multiple recurrences, followup-scans, CA-125. [Gynecological oncologist and medical oncologist] opinions differ."

Participant C: "Leg pain from [carboplatin/taxol] still present. Issues with my sleep. 6 months post chemo. Told leg pain was a chemo gift and would stay."

Survivors reported managing their illness by maintaining a sense of self and engaging in healthy behaviors such as being physically active, eating a healthy diet, and reducing stress. One survivor noted that she has become a research advocate and provides peer support to other ovarian cancer patients.

Participant B: "Serve ovarian cancer community as research advocate and provide peer support."

When asked about how their health care providers addressed the topic of whether or not they had received a care plan when ending treatment, survivors reported a low amount of support. Only one survivor said she had received a survivorship care plan when she ended her treatment. While many survivors noted that they had become involved in advocacy organizations, peer support groups, or both, they simultaneously noted needing personal space to "take frequent breaks from being all teal [the ribbon color for ovarian cancer] to being just me" (Participant E). One survivor reported feeling like a "rudderless ship" (Participant A) after treatment ended because of the high number of sensitive, less-clearly addressed issues she faced without anyone to steer or guide her along the way. On the other hand, one woman (Participant E) described the posttreatment phase as being a time when she was recapturing and redefining her identity: "Free to be me again despite history of cancer or fears!"

\section{DISCUSSION}

It is unsurprising yet confirmatory that survivors and stakeholders alike agreed that ovarian cancer survivors receive little support as they transition into the survivorship period of their cancer experience. Although women reported ongoing physical and emotional needs that were largely unaddressed by their providers, they continued to find ways to manage their health and maintain a sense of self. Given that so many women will recur and will continue to experience severe and distressing symptoms after treatment is over, this gap in care should serve as a call to action. In this Twitter chat, survivors noted that they wanted their trusted health care professionals to proactively equip them with the supportive care resources they will need but may not have the means or regular clinic appointments with their health care team to subsequently request.

Survivorship care plans primarily emphasize the supportive care of patients who complete curative intent treatment and for whom there is a high probability of long-term survival. ${ }^{7}$ These plans aim to help this population receive evidence-based, coordinated care. Key to patients' long-term health, survivorship care plans help bridge oncology and primary care. One study demonstrated that when oncologists share survivorship care plans with a patient's primary care provider, the primary care provider is 9 times more likely to discuss survivorship issues with the patient; yet, the unclear delineation of patient care responsibilities and lack of training in oncology and survivorship care remain barriers to primary care providers delivering follow-up care to survivors. ${ }^{15}$ Moreover, most patients with ovarian cancer prefer to receive continued care from their oncologist. ${ }^{16}$

The Society of Gynecologic Oncology has published its version of a survivorship care plan, called the Survivorship Toolkit, which contains resources aimed for patients completing therapy. ${ }^{17}$ However, as with other survivorship care plans, it assumes all patients who require a survivorship care plan are being treated with curative intent and does not address the needs of patients who eventually relapse. ${ }^{18}$ Furthermore, it does not address the totality of patient needs beyond medical care, such as the emotional issues and coping discussed by patients in this study and the evaluation and treatment for pelvic/lower-extremity lymphedema, genitourinary symptoms of menopause, and sexual health. As a result, there is very little guidance as to 
what survivorship care should look like for women who face a high risk of recurrence, those who recur, and those with advanced or metastatic disease who may not "complete" curative intent therapy.

Our finding that few patients received survivorship care plans despite facing ongoing needs reflects the lack of effective survivorship care models that fulfill the Institute of Medicine's 2006 recommendation."7 Nonetheless, the Commission on Cancer is mandating the implementation of survivorship care plans within its accredited hospitals, ${ }^{19}$ and research demonstrating how to do so within ovarian cancer is desperately needed. In one of the few trials that looked at women with a gynecologic cancer, Nicolaije et $\mathrm{al}^{20}$ reported results of a randomized controlled trial that compared an automatically generated survivorship care plan to usual care among women with endometrial cancer. While $74 \%$ of patients randomly assigned to receive a survivorship care plan actually received one, there was no difference in satisfaction with either information or care compared to those in the usual care arm. Of concern, survivorship care plans were associated with elevated levels of patient concerns, emotional impact, and symptoms. Similarly, a randomized clinical trial reported by de Rooij et al found that, compared to women with ovarian cancer who did not receive a survivorship care plan, those who did receive a plan reported no differences in satisfaction and had lower beliefs that treatment would help cure their disease. ${ }^{21}$

A current trial (NCT03035773) sponsored by Sidney Kimmel Comprehensive Cancer Center in collaboration with Patient-Centered Outcomes Research Institute and guided by a stakeholder advisory board is evaluating three models of survivorship care planning to determine which model is feasible, effective, and patient-centered. While this study will not recruit women with gynecologic cancer, these prospective studies evaluating patient-centered survivorship care have direct implications for the care of patients with ovarian cancer and similar studies can be implemented within the ovarian cancer population.

Future research should focus on how health care providers and cancer advocacy organizations can continue to support women with ovarian cancer after they end first-line treatment. In fact, the National Academies of Sciences, Engineering, and Medicine emphasized the need for prospective studies that are specific to ovarian cancer; appreciate differences in patient age, race, and ethnicity; and reveal risk factors for specific issues in survivorship. ${ }^{22}$ Understanding their needs and providing continuous support can ensure that survivors do not feel like they are being abandoned after treatment, living in uncertainty and fear. Likewise, research should consider the needs of patients with ovarian cancer who recur and those who require continuous treatment, since these survivors need assistance living with the disease rather than living after the disease. We must find ways to provide care plans and support systems that proactively address the emotional and physical concerns so prevalent within this patient population.

As researchers continue to identify and test the efficient, clinically beneficial ways to support ovarian cancer survivors, clinicians must continue to review and enact evidence-based standards for survivorship care. For example, the Society of Gynecologic Oncology published recommendations in 2011, with an update in 2017, for the posttreatment care for survivors of gynecologic cancer, including ovarian cancer. ${ }^{23,24}$ Clinicians can use survivorship care guidelines set by the American Society of Clinical Oncology and other national cancer organizations to address the multitude of ongoing physical and psychosocial symptoms faced by many cancer survivors. ${ }^{25}$

\section{Limitations}

Compared to traditional focus groups, Twitter chats offer distinct benefits and challenges. We were able to access a wide-ranging group of participants from across the United States who self-identified as being interested in this topic. However, our sample did not include ovarian cancer survivors who do not have Twitter accounts or were not comfortable using social media to share information and opinions regarding their cancer care. Also, given the heterogeneity of our sample and the short window in which questions were asked, participants may or may not have felt comfortable building off of each other's comments, as is typically done during a focus group. Another limitation is the bias in attracting participants who already engage in social media and internet support. While the richness of other qualitative methodologies may be lacking, the ability to quickly capture the central themes of survivors' experiences makes Twitter chats an attractive qualitative method. 


\section{CONCLUSIONS}

Results of this Twitter chat suggest that ovarian cancer survivors receive minimal information to guide their health and well-being during the survivorship period. Health care providers must prepare these patients as they end treatment for the physical, psychological, and social needs we know they will likely face. Survivorship care plans must be tailored to meet the unique concerns and cancer experience of this patient population. The health care and advocacy communities must reach out to these patients as they transition from active treatment to surveillance. This vulnerable time in patients' lives is our opportunity to demonstrate our understanding of their needs and concerns.

\section{Patient-Friendly Recap}

- Due to high rates of recurrence, patients with ovarian cancer require long-term supportive health care.

- The authors recruited ovarian cancer survivors to participate in a moderated Twitter chat to discuss their experiences after treatment.

- Contrary to the Commission on Cancer's recommendations, few survivors receive detailed care plans when their treatment ends.

- Survivors expressed a desire for health providers to proactively offer resources that could help address future emotional and physical needs.

\section{Author Contributions}

Study design: all authors. Data acquisition or analysis: Hagan Thomas, Nauth-Shelley, Sparacio, Lizaso, Utengen, Dizon. Manuscript drafting: Hagan Thomas. Critical revision: all authors.

\section{Conflicts of Interest}

None.

\section{References}

1. Ferrell B, Smith S, Cullinane C, Melancon C. Symptom concerns of women with ovarian cancer. J Pain Symptom Manage. 2013;25:528-38. CrossRef

2. Ozga M, Aghajanian C, Myers-Virtue S, et al. A systematic review of ovarian cancer and fear of recurrence. Palliat Support Care. 2015;13:1771-80. CrossRef

3. American Cancer Society. Cancer Facts \& Figures 2017. https://www.cancer.org/content/dam/cancer-org/research/ cancer-facts-and-statistics/annual-cancer-facts-andfigures/2017/cancer-facts-and-figures-2017.pdf. Atlanta, GA: American Cancer Society, 2017.
4. Ahmed-Lecheheb D, Joly F. Ovarian cancer survivors' quality of life: a systematic review. J Cancer Surviv. 2016;10:789-801. CrossRef

5. Gonçalves V. Long-term quality of life in gynecological cancer survivors. Curr Opin Obstet Gynecol. 2010;22:30-5. CrossRef

6. Mirabeau-Beale KL, Kornblith AB, Penson, RT, et al. Comparison of the quality of life of early and advanced stage ovarian cancer survivors. Gynecol Oncol. 2009;114:353-9. CrossRef

7. Committee on Cancer Survivorship: Improving Care and Quality of Life; National Cancer Policy Board; Hewitt M, Greenfield S, Stovall E (eds). From Cancer Patient to Cancer Survivor: Lost in Transition. Washington DC, National Academies Press, 2006.

8. Mayer DK, Green M, Check DK, et al. Is there a role for survivorship care plans in advanced cancer? Support Care Cancer. 2015;23:2225-30. CrossRef

9. Dizon DS, Schutzer ME, Politi MC, Linkletter CD, Miller SC, Clark MA. Advance care planning decisions of women with cancer: provider recognition and stability of choices. J Psychosoc Oncol. 2009;20;27:383-95. CrossRef

10. Urbaniec OA, Collins K, Denson LA, Whitford HS. Gynecological cancer survivors: assessment of psychological distress and unmet supportive care needs. J Psychosoc Oncol. 2011;29:534-51. CrossRef

11. Roland KB, Rodriguez JL, Patterson JR, Trivers KF. A literature review of the social and psychological needs of ovarian cancer survivors. Psychooncology. 2013;22:2408-18. CrossRef

12. Attai DJ, Sedrak MS, Katz MS, et al. Social media in cancer care: highlights, challenges \& opportunities. Future Oncol. 2016;12:1549-52. CrossRef

13. Creswell JW. Qualitative Inquiry and Research Design: Choosing Among Five Approaches, Second Edition. Thousand Oaks, CA: Sage Publications, 2007, pp. 148-55.

14. Moreno MA, Goniu N, Moreno PS, Diekema D. Ethics of social media research: common concerns and practical considerations. Cyberpsychol Behav Soc Netw. 2013;16:708-13. CrossRef

15. Blanch-Hartigan D, Forsythe LP, Alfano CM, et al. Provision and discussion of survivorship care plans among cancer survivors: results of a nationally representative survey of oncologists and primary care physicians. $J$ Clin Oncol. 2014;32:1578-85. CrossRef

16. Mayer DK, Nekhlyudov L, Snyder CF, et al. American Society of Clinical Oncology clinical expert statement on cancer survivorship care planning. J Clin Oncol. 2014;10:345-51. CrossRef

17. Weaver KE, Aziz NM, Arora NK, et al. Follow-up care experiences and perceived quality of care among long-term survivors of breast, prostate, colorectal, and gynecologic cancers. J Clin Oncol. 2014;10:e231-9. CrossRef

18. Society of Gynecologic Oncology. Survivorship toolkit. https://www.sgo.org/clinical-practice/management/ survivorship-toolkit/. Accessed October 16, 2017.

19. Commission on Cancer. Cancer Program Standards 2012: Ensuring Patient-Centered Care, V1.2.1. https://www. facs.org/ /media/files/quality\%20programs/cancer/coc/ programstandards2012.ashx. Chicago, IL: American College of Surgeons, 2012.

20. Nicolaije KA, Husson O, Ezendam NP, et al. Endometrial cancer survivors are unsatisfied with received information about diagnosis, treatment and follow-up: a study from the population-based PROFILES registry. Patient Educ Couns. 2012;88:427-35. CrossRef 
21. de Rooij BH, Ezendam NPM, Nicolaije KAH, et al. Effects of Survivorship Care Plans on patient reported outcomes in ovarian cancer during 2-year follow-up - The ROGY care trial. Gynecol Oncol. 2017;145:319-28. CrossRef

22. Committee on the State of the Science in Ovarian Cancer Research; Board on Health Care Services; Institute of Medicine; National Academies of Sciences, Engineering, and Medicine. Ovarian Cancers: Evolving Paradigms in Research and Care. Washington, DC: National Academies Press, 2016, pp. 237-8.

23. Salani R, Backes FJ, Fung MF, et al. Posttreatment surveillance and diagnosis of recurrence in women with gynecologic malignancies: Society of Gynecologic Oncologists recommendations. Am J Obstet Gynecol. 2011;204:466-78. CrossRef
24. Salani R, Khanna N, Frimer M, Bristow RE, Chen LM. An update on post-treatment surveillance and diagnosis of recurrence in women with gynecologic malignancies: Society of Gynecologic Oncology (SGO) recommendations. Gynecol Oncol. 2017;146:3-10. CrossRef

25. American Society of Clinical Oncology. Guidelines on survivorship care. https://www.asco.org/practiceguidelines/cancer-care-initiatives/prevention-survivorship/ survivorship-compendium-0. Accessed October 16, 2017.

(C) 2018 Aurora Health Care, Inc. 